\title{
Measurement and Modeling of Temporal and Spatial Indoor Multipath Characteristics
}

Michael A. Jensen*, Quentin H. Spencer, A. Lee Swindlehurst, and Brian D. Jeffs Department of Electrical and Computer Engineering Brigham Young University Provo, UT 84602

I. Introduction Wideband digital wireless communications are becoming increasingly practical in indoor environments. For example, the availability of new frequency spectra in the $900 \mathrm{MHz}, 2.4 \mathrm{GHz}$, and $5.7 \mathrm{GHz}$ ranges has fostered the development of systems for local area networks. One of the limitations associated with such networks stems from the challenging indoor propagation environment, which leads to multipath signals with significant temporal and angular spreading. This multipath results in intersymbol interference (ISI) that can seriously reduce the achievable transmission data rate.

One important approach for reducing ISI is the use of "smart" antennas capable of adapting the antenna response. However, in order to assess the performance of different array configurations and algorithms, it is necessary to have a detailed understanding of the channel the temporal and spatial multipath characteristics. While previous models have been formulated for multipath temporal properties [1] and some measurements have been presented for spatial characteristics [2], there has not been a campaign aimed at developing a joint time-space model using measured responses. This paper presents the results of such an effort.

II. Data Acquisition Detailed characterization of the temporal and spatial multipath structure mandates a relatively sophisticated data collection campaign [2]. Figure 1 shows the platform used to accomplish these measurements. The system used an HP $8720 B$ network analyzer to measure the channel transmission characteristics between 6.75 and $7.25 \mathrm{GHz}$ and transform the results to provide the time-domain response with a resolution of roughly $3 \mathrm{~ns}$. Physical remoting of the transmit antenna was accomplished using a $36 \mathrm{~m}$ length of coaxial cable. The 60 $\mathrm{cm}$ parabolic reflector used to collect the multipath signal offered a $3 \mathrm{~dB}$ beamwidth (resolution) of $6^{\circ}$. The collected data was processed using the CLEAN algorithm in conjunction with measured spatial and temporal point response functions for the data collection system. This processing produced the time and angle of arrival for each individual multipath component.

Data for this campaign was collected in two different engineering buildings on the Brigham Young University campus. 55 data sets were recorded in the Clyde Building (CB) which consists of steel-reinforced concrete structural walls and cinder-block partition walls. 10 additional data sets were collected in the Crabtree building (CTB), which is constructed of steel-reinforced concrete structural walls and gypsum board/steel stud partition walls.

III. Signal Statistics The measured multipath characteristics can be used to generate a statistical model for the propagation environment [1]. To formulate this model, we first observe that arrivals appear in groups or "clusters" in both space and time $\{1\}$. Each cluster is characterized by a delay $T$ and arrival angle $\Theta$. Individual arrivals or "rays" within each cluster each possess a delay $\tau$, arrival angle $\omega$, 0-7803-5639-X/99/\$10.00 @1999 IEEE 
and amplitude $\beta$. Mathematically, this model for the channel impulse response can be written as

$$
h(t, \theta)=\sum_{\ell=0}^{\infty} \sum_{k=0}^{\infty} \beta_{k \ell} e^{j \phi_{k \ell}} \delta\left(t-T_{\ell}-\tau_{k \ell}\right) \delta\left(\theta-\Theta_{\ell}-\omega_{k \ell}\right)
$$

where the sums over $\ell$ and $k$ represent clusters and arrivals within each cluster respectively. The phase term $\phi_{k \ell}$ is assumed to be uniformly distributed [1]. It should be noted that the cluster arrival times and angles are taken with reference to values for the first arrival $\left(T_{0}\right.$ and $\left.\Theta_{0}\right)$ to make the measured parameters independent of the room geometry and measurement range. Additionally, all amplitudes are normalized to that of the initial arrival.

Figure 2 provides scatter plots of the arrival times and angles for clusters and individual arrivals within clusters for all of the collected data. These figures show that there is no significant correlation between time and angle in either case. Based upon this observation, we assume that the times and angles of arrival are independent. Figure 3 illustrates plots of the normalized cluster and ray amplitudes versus relative delay for the CB. There is significant variance in these plots, although the mean square value roughly follows an exponential curve (solid curve in plots). Given this observation, we utilize the findings of [1] which model the amplitude as Rayleigh distributed with a double exponentially decaying mean square value or

$$
\overline{\beta_{k \ell}^{2}}=\overline{\beta_{00}^{2}} e^{-\tau_{l} / \Psi} e^{-\tau_{k \ell} / \psi} \text {. }
$$

Figure 4 presents the cumulative distribution function (CDF) for the cluster and ray arrival times in the $C B$. Again, we adopt the model of [1] which assumes these arrival times are modeled as Poisson distributed random variables conditioned on the time of arrival of the previous cluster or ray. These probability density functions (PDF) may be expressed as

$$
p\left(T_{\ell} \mid T_{\ell-1}\right)=\Lambda e^{-\Lambda\left(T_{\ell}-T_{\ell-1}\right)} \quad p\left(\tau_{k \ell} \mid \tau_{(k-1) \ell}\right)=\lambda e^{-\lambda\left(\tau_{k \ell}-\tau_{(k-1) \ell}\right)}
$$

where $\Lambda$ and $\lambda$ represent the cluster and ray arrival rates, respectively. The best fitting CDF for each parameter is shown in Figure 4.

Finally, Figure 5(a) shows the CDF for the cluster arrival angles in the CB. This data suggests that the distribution on $\Theta$ is relatively uniform on $0^{\circ}$ to $360^{\circ}$. Figure 5 (b) shows the PDF for the ray arrival angles relative to the mean arrival angle of the cluster. Superimposed on this PDF is the best fitting zero-mean Laplacian distribution expressed as

$$
p\left(\omega_{k \ell}\right)=e^{-\left|\sqrt{2} \omega_{k \ell} / \sigma\right|} / \sqrt{2} \sigma
$$

Table 1 lists the different parameters of this model for the two different buildings examined in this study. As can be seen, the results describing the temporal behavior are noticeably dissimilar, likely due to the different constructions in the building interiors. However, the arrival angle information is strikingly similar.

IV. Conclusions We have presented measurements and models of the temporal and spatial characteristics of the multipath indoor wireless channel. The measurement scheme uses a directional antenna in conjunction with a wideband probing signal 
Table 1: A comparison of model parameters for the two buildings used in this study.

\begin{tabular}{lcccccc}
\hline \hline Building & $\Psi$ & $\psi$ & $1 / \Lambda$ & $1 / \lambda$ & $\sigma$ \\
\hline $\mathrm{CB}$ & $34 \mathrm{~ns}$ & $29 \mathrm{~ns}$ & $17 \mathrm{~ns}$ & $5 \mathrm{~ns}$ & $26^{\circ}$ \\
$\mathrm{CTB}$ & $78 \mathrm{~ns}$ & $82 \mathrm{~ns}$ & $17 \mathrm{~ns}$ & $7 \mathrm{~ns}$ & $22^{\circ}$ \\
\hline
\end{tabular}

The data are used to develop a simple statistical model and determine appropriate parameter models. Current work is being performed to explore channel properties at other frequencies such as in the $2.4 \mathrm{GHz}$ and $5.7 \mathrm{GHz}$ bands. Studies of the performance benefits of using more sophisticated models are also underway.

\section{REFERENCES}

[1] A. A. M. Saleh and R. A. Valenzuela, IEEE J. Selected Areas Commun., SAC5, pp. 128-137, 1987.

[2] J. Litva, A. Ghaforian, and V. Kezys, Proc. 1996 IEEE AP-S Intl. Symp., July 21-26, Baltimore, MD, 2, pp. 1490-1493, 1996.

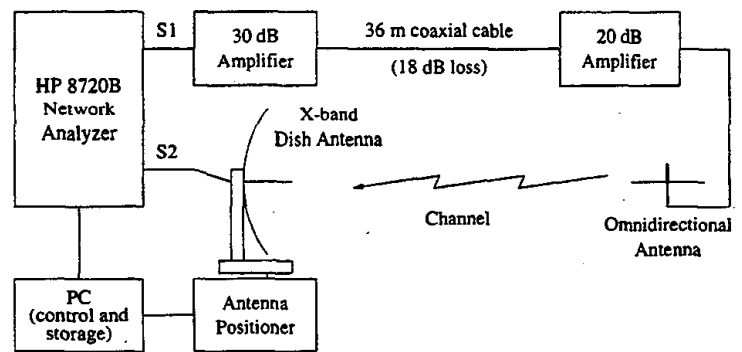

Figure 1: The data acquisition system used to collect time-space multipath data.

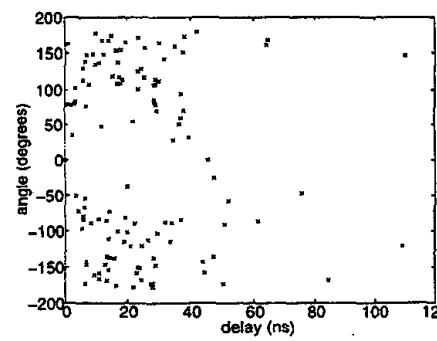

(a)

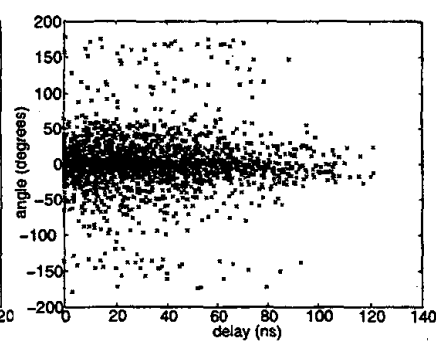

(b)

Figure 2: Scatter plot of arrival time and angle for (a) clusters and (b) rays within clusters. 


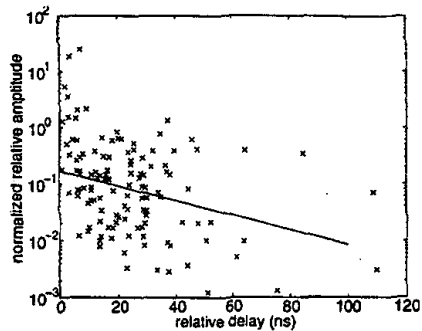

(a)

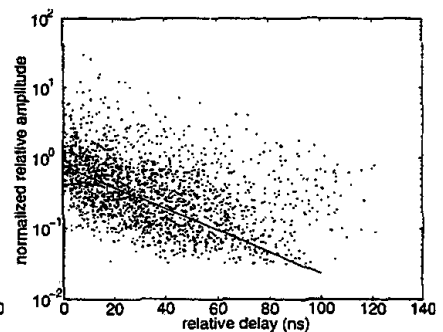

(b)

Figure 3: Normalized (a) cluster and (b) ray amplitudes versus relative delay for the CB with best fitting exponential curves shown.

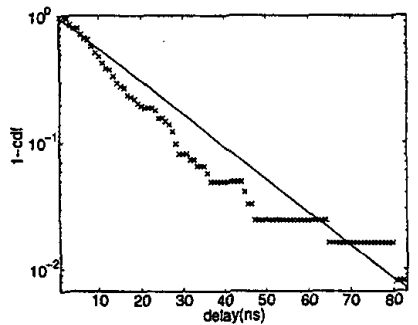

(a)

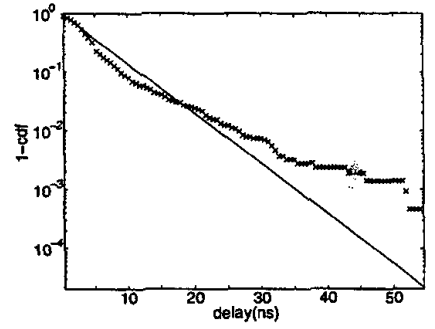

(b)

Figure 4: CDF of relative arrival times for (a) clusters and (b) rays within clusters with the best fitting Poisson PDF shown.

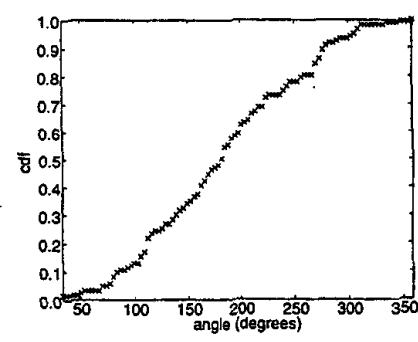

(a)

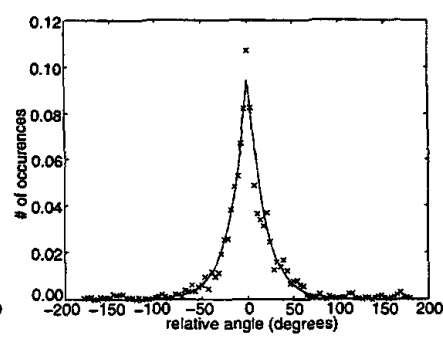

(b)

Figure 5: (a) CDF of mean cluster angles with respect to the first cluster in each set and (b) PDF of ray angles relative to each cluster mean for the CB. 